\title{
A Hierarchy of Unary Primitive Recursive String-functions
}

\author{
Lila Santean* \\ Institute for Informatics \\ 8-10 Miciurin blvd. \\ 71316 Bucharest 1, Romania
}

\begin{abstract}
Using a recent result of G.Asser, an extention of Ackermann-Peter hierarchy of unary primitive recursive functions to string-functions is obtained. The resulting hierarchy classifies the string-functions according to their lexicographical growth.
\end{abstract}

\section{Introduction}

Let $\mathbf{N}$ be the set of naturals i.e. $\mathbf{N}=\{0,1,2, \ldots\}$. Consider a fixed alphabet $A=\left\{a_{1}, a_{2}, \ldots, a_{r}\right\}, r \geq 2$ and denote by $A^{*}$ the free monoid generated by $A$ under concatenation (with $e$ the null string). The elements of $A^{*}$ are called strings; if reffering to strings, " $<$ " denotes the lexicographical order induced by $a_{1}<a_{2}<\ldots<a_{r}$. Denote by $F n c$ (respectively $F n c_{A}$ ) the set of all unary number-theoretical (respectively, string) functions. By $I, S u c c, C_{m}, P d$ we denote the following number-theoretical functions:

$$
\begin{aligned}
I(x)= & x, \\
\operatorname{Succ}(x)= & x+1, \\
C_{m}(x)= & m, \\
\operatorname{Pd}(x)= & x-1, \text { where } x-y=\max \{x-y, 0\}, \\
& \text { for all } x, m, y \in \mathbf{N} .
\end{aligned}
$$

By $I^{A}, S u c c_{i}^{A}, C_{u}^{A}, \sigma, \pi$, we denote the following string-functions:

$$
I^{A}(w)=w,
$$

*Present address: Department of Mathematics, University of Turku, 20500 Turku, Finland 


$$
\begin{aligned}
& \operatorname{Succ}_{i}^{A}(w)=w a_{i}(1 \leq i \leq r), \\
& C_{u}^{A}(w)=u, \\
& \sigma(e)=a_{1}, \sigma\left(w a_{i}\right)=w a_{i+1} \text { if } 1 \leq i<r \text { and } \sigma\left(w a_{r}\right)=\sigma(w) a_{1} \\
& \pi(e)=e, \pi(\sigma(w))=w \\
& \text { for all } w, u \in A^{*}
\end{aligned}
$$

Furtheron one uses the primitive recursive bijections $c: A^{*} \longrightarrow \mathbf{N}, \bar{c}: \mathbf{N} \longrightarrow A^{*}$ given by

$$
\begin{aligned}
& c(e)=0, c\left(w a_{i}\right)=r \cdot c(w)+i, 1 \leq i \leq r, w \in A^{*} \\
& \bar{c}(0)=e, \bar{c}(m+1)=\sigma(\bar{c}(m)), m \in \mathbf{N} .
\end{aligned}
$$

To each $f$ in $F n c$ one associates the string-function $s(f) \in F n c_{A}$ defined by $s(f)(w)=\bar{c}(f(c(w)))$ and for each $g$ in $F n c_{A}$ one associates the numbertheoretical function $n(g)$ defined by $n(g)(x)=c(g(\bar{c}(x)))$. It is easily seen that for every string-function $g, s(n(g))=g$ and for every number-theoretical function $f, n(s(f))=f$. For example, $s($ Succ $)=\sigma, n\left(I^{A}\right)=I, s(P d)=\pi$. A mapping from $F_{n c^{n}}$ to $F_{n c}$ is called an operator in $F n c$, and analogously for $F n c_{A}$. We consider the following operators in $F n c$ and $F n c_{A}$ :

$$
\begin{aligned}
\operatorname{sub}(f, g) & =h \Longleftrightarrow f, g, h \in F n c, f(g(x))=h(x) \\
\operatorname{diff}_{(f, g)} & =h \Longleftrightarrow f, g, h \in F n c, h(x)=f(x)-g(x) \\
i t_{x}(f) & =h \Longleftrightarrow f, h \in F_{n c}, h(0)=x, h(y+1)=f(h(y)) \\
\operatorname{sub}_{A}(f, g) & =h \Longleftrightarrow f, g, h \in F_{n c_{A}}, f(g(w))=h(w) ; \\
\sigma-i t_{A, w}(f) & =h \Longleftrightarrow f, h \in F_{n c_{A}}, h(e)=w, h(\sigma(u))=f(h(u)) .
\end{aligned}
$$

For every operator $\varphi$ in $F n c, s(\varphi)(f)=s(\varphi(n(f)))$, for every $f \in F n c$; analogously, for every operator $\theta$ in $F n c_{A}, n(\theta)(g)=n(\theta(s(g)))$, for every $g \in F n c$. For example, $s\left(i t_{x}\right)=\sigma-i t_{A, c(x)}, n\left(\sigma-i t_{A, w}\right)=i t_{\bar{c}(w)}$.

\section{Ackermann-Peter string-function}

The primitive-recursive functions were introduced by Asser [1] and studied by various authors (see [4], [6], [8]). In order to study the complexity of such functions, we use as a measure of complexity the growth relatively to the lexicographical order. To this aim we use the string-version of the Ackermann-Peter unary function defined by Weichrauch [8]. The function, denoted by $A: A^{*} \longrightarrow A^{*}$, is given by means of the following three equations :

$$
\begin{aligned}
A_{0}(x) & =\sigma(x) \\
A_{n+1}(e) & =A_{n}\left(a_{1}\right) \\
A_{n+1}(\sigma(x)) & =A_{n}\left(A_{n+1}(x)\right) .
\end{aligned}
$$

The following technical results concern the monotonicity properties of the function $A$; they generalize the monotonicity properties of the number-theoretical Ackermann-Peter function (see [4]). 
Lemma 1 For all naturals $n$ and for all strings $x$ over $A^{*}$, we have

$$
A_{n}(x)>x \text {. }
$$

Proof. We proceed by induction on $n$.

For $n=0$ we have $A_{0}(x)=\sigma(x)>x$. We assume that $A_{n}(x)>x$ and we prove the inequality $A_{n+1}(x)>x$ by induction on $x$.

For $x=e, A_{n+1}(e)=A_{n}\left(a_{1}\right)>e$. Suppose now that $A_{n+1}(x)>x$. We use (3) and the first induction hypothesis to get

$$
A_{n+1}(\sigma(x))=A_{n}\left(A_{n+1}(x)\right)>A_{n+1}(x) .
$$

Finally, by the second induction hypothesis, that is $A_{n+1}(x) \geq \sigma(x)$, we obtain $A_{n+1}(\sigma(x))>\sigma(x)$.

Lemma 2 For all naturals $n$ and for all strings $x$ over $A^{*}$, we have:

$$
A_{n}(x)<A_{n}(\sigma(x)) \text {. }
$$

Proof. For $n=0$,

$$
A_{0}(x)=\sigma(x)<\sigma(\sigma(x))=A_{0}(\sigma(x)) .
$$

Assume that $A_{n}(x)<A_{n}(\sigma(x))$. In view of (3) and lemma 1 we have

$$
A_{n+1}(\sigma(x))=A_{n}\left(A_{n+1}(x)\right)>A_{n+1}(x) .
$$

Corollary 1 For all naturals $n$ and all strings $x, y$ from $A^{*}$, if $x<y$, then $A_{n}(x)<A_{n}(y)$.

Lemma 3 For all naturals $n$ and for all strings $x$ over $A^{*}$, we have

$$
A_{n}(x)<A_{n+1}(x) \text {. }
$$

Proof. We proceed by double induction on $n$ and $x$.

For $n=0$ we have

$$
A_{0}(x)=\sigma(x)<\sigma(\sigma(x))=A_{1}(x) .
$$

Assume now that $A_{n}(x)<A_{n+1}(x)$ and we prove that $A_{n+1}(x)<A_{n+2}(x)$ by induction on $x$.

For $x=e$, in view of (2) and the first induction hypothesis, we get

$$
A_{n+1}(e)=A_{n}\left(a_{1}\right)<A_{n+1}\left(a_{1}\right)=A_{n+2}(e) .
$$

In view of a new induction hypothesis, $A_{n+1}(x)<A_{n+2}(x)$, we deduce the relations:

$$
A_{n+1}(\sigma(x))=A_{n}\left(A_{n+1}(x)\right)<A_{n}\left(A_{n+2}(x)\right)<A_{n+1}\left(A_{n+2}(x)\right)=A_{n+2}(\sigma(x))
$$

(we have also used the first induction hypothesis, relation (3) and corollary 1). 
Corollary 2 For all naturals $n$ and $m$, and for all strings $x$ in $A^{*}$, if $n<m$, then

$$
A_{n}(x)<A_{m}(x)
$$

Lemma 4 For all strings $x$ of $A^{*}$ we have: $A_{2}(x)=\sigma^{2 c(x)+3}(e)$.

Proof. We proceed by induction on $x$.

For $x=e$, in view of (2) we have

$$
A_{2}(e)=A_{1}\left(a_{1}\right)=\sigma\left(\sigma\left(a_{1}\right)\right)=\sigma^{3}(e)=\sigma^{2 c(e)+3}(e) .
$$

Assuming that $A_{2}(x)=\sigma^{2 c(x)+3}(e)$, we prove that $A_{2}(\sigma(x))=\sigma^{2 c(\sigma(x))+3}(e)$. Indeed, using (3) and the equality $c(\sigma(x))=c(x)+1$, we get:

$$
A_{2}(\sigma(x))=A_{1}\left(A_{2}(x)\right)=A_{1}\left(\sigma^{2 c(x)+3}(e)\right)=\sigma^{2 c(x)+5}(e)=\sigma^{2 c(\sigma(x))+3}(e) .
$$

Lemma 5 For all naturals $k$ and $n \geq 1$, there exists a natural $i$ (which depends upon $k$ ) such that

$$
A_{n}\left(\sigma^{k}(x)\right)<A_{n+1}\left(\pi^{k}(x)\right),
$$

for every string $x$ in $A^{*}$ with $c(x)>i$.

Proof. We first notice that for every string $x$ with $c(x)>3 k-1$, we have $\sigma^{k}(x)<A_{2}\left(\pi^{k+1}(x)\right)$.

Indeed, by lemma 4 we have

$$
\begin{aligned}
A_{2}\left(\pi^{k+1}(x)\right) & =\sigma^{2 c\left(\pi^{k+1}(x)\right)+3}(e)=\sigma^{2(c(x)-k \dot{-1})+3}(e)=\sigma^{2 c(x)-2 k+1}(e) \\
& >\sigma^{k+c(x)}(e)=\sigma^{k}\left(\sigma^{c(x)}(e)\right)=\sigma^{k}(x) .
\end{aligned}
$$

Consequently, using corolary 1 and corollary 2,

$$
A_{n}\left(\sigma^{k}(x)\right)<A_{n}\left(A_{2}\left(\pi^{k+1}(x)\right)\right)<A_{n}\left(A_{n+1}\left(\pi^{k+1}(x)\right)\right)=A_{n+1}\left(\pi^{k}(x)\right),
$$

for all strings $x$ with $c(x)>3 k-1$. In conclusion, we can take $i=3 k-1$.

Lemma 6 For all naturals $n$ and strings $x$ in $A^{*}$ we have

$$
A_{n+1}(x)=A_{n}^{c(x)+1}\left(a_{1}\right) \text {. }
$$

Proof. We proceed by induction on $x$.

For $x=e$, using (2) we obtain

$$
A_{n+1}(e)=A_{n}\left(a_{1}\right)=A_{n}^{c(e)+1}\left(a_{1}\right) .
$$

Assuming that $A_{n+1}(x)=A_{n}^{c(x)+1}\left(a_{1}\right)$ we prove the equality

$$
A_{n+1}(\sigma(x))=A_{n}^{c(\sigma(x))+1}\left(a_{1}\right) \text {. }
$$

Indeed, using (3) we get:

$A_{n}^{c(\sigma(x))+1}\left(a_{1}\right)=A_{n}^{c(x)+2}\left(a_{1}\right)=A_{n}\left(A_{n}^{c(x)+1}\left(a_{1}\right)\right)=A_{n}\left(A_{n+1}(x)\right)=A_{n+1}(\sigma(x))$.

The monotonicity properties of the string Ackermann-Peter function will be freely used in what follows. 


\section{A hierarchy of unary primitive recursive string-functions}

We are going to define an increasing sequence $\left(C_{n}\right)_{n \geq 0}$ of string-function classes whose union equals the class of the one-argument primitive recursive stringfunctions.

Definition 1 We say that the function $f: A^{*} \longrightarrow A^{*}$ is defined by limited iteration at $e$ (shortly, limited iteration) from the functions $g: A^{*} \longrightarrow A^{*}$ and $h: A^{*} \longrightarrow A^{*}$ if it satisfies the following equations:

$$
\begin{aligned}
f(e) & =e \\
f(\sigma(x)) & =g(f(x)), \\
f(x) & \leq h(x),
\end{aligned}
$$

for every $x$ in $A^{*}$.

Definition 2 For a natural $n$ we define $C_{n}$ to be the smallest class of unary primitive recursive string-functions which contains the functions $A_{0}, A_{n}$ and is closed under composition, limited iteration and $s($ diff) (the string-function operation corresponding to the arithmetical difference).

Lemma 7 For all naturals $n$, the class $C_{n}$ contains the functions $C_{e}^{A}, I^{A}, \pi$ and the functions $l_{i}(1 \leq i \leq r)$, sg and $\overline{s g}$ defined by:

$$
\begin{aligned}
l_{i}(w)= & a_{i}, 1 \leq i \leq r, \\
s g(w)= & \begin{cases}e & \text { if } w=e \\
a_{1} & \text { if } w \neq e\end{cases} \\
\overline{s g}(w)= & \begin{cases}a_{1} & \text { if } w=e \\
e & \text { if } w \neq e,\end{cases} \\
& \text { for all } w \in A^{*} .
\end{aligned}
$$

Proof. It follows from the following equalities:

$$
\begin{aligned}
C_{e}^{A} & =s(\text { diff })\left(A_{0}, A_{0}\right) \\
l_{i} & =A_{0}^{i}(e), 1 \leq i \leq r \\
I^{A} & =s(\text { diff })\left(A_{0}, l_{1}\right) \\
\overline{s g} & =s(\text { diff })\left(l_{1}, I^{A}\right) \\
s g & =s(\text { diff })\left(l_{1}, \overline{s g}\right) \\
\pi & =s(\text { diff })\left(I^{A}, l_{1}\right)
\end{aligned}
$$

and from the definition 2 .

Theorem 1 For all naturals $n, C_{n} \subseteq C_{n+1}$. 
Proof. We shall prove by induction on $n$ that for all natural numbers $n$ and $k, A_{n} \in C_{n+k}$.

If $n=0$, by definition $2, A_{0} \in C_{m}$, for every natural $m$. Assume that $A_{n} \in C_{n+k}, \forall k \in \mathbf{N}$. We shall prove that $A_{n+1} \in C_{n+k+1}, \forall k \in \mathbf{N}$.

Assertion: For every string $x, A_{n+1}(x)=f(\sigma(x))$, where

$$
\begin{aligned}
f(e) & =e, \\
f(\sigma(x)) & =A_{n}(g(f(x))), \text { and } \\
g(x) & =s(\operatorname{diff})(\sigma(x), s g(x)) .
\end{aligned}
$$

The equalities will be proved by induction on the string $x$. If $x=e$, from the definitions of the functions $A_{n}$ and $s$ (diff) we deduce:

$$
\begin{aligned}
f(\sigma(e)) & =A_{n}(g(f(e)))=A_{n}(g(e))=A_{n}(s(\text { diff })(\sigma(e), s g(e))) \\
& =A_{n}\left(s(\text { diff })\left(a_{1}, e\right)\right)=A_{n}\left(a_{1}\right)=A_{n+1}(e) .
\end{aligned}
$$

Supposing now that $A_{n+1}(x)=f(\sigma(x))$, we shall show that $A_{n+1}(\sigma(x))=$ $f\left(\sigma^{2}(x)\right)$.

Indeed,

$$
\begin{aligned}
f(\sigma(\sigma(x))) & =A_{n}(g(f(\sigma(x))))=A_{n}\left(g\left(A_{n+1}(x)\right)\right) \\
& =A_{n}\left(s(\operatorname{diff})\left(\sigma\left(A_{n+1}(x)\right), s g\left(A_{n+1}(x)\right)\right)\right) \\
& =A_{n}\left(s(\operatorname{diff})\left(\sigma\left(A_{n+1}(x)\right), a_{1}\right)\right) \\
& =A_{n}\left(\bar{c}\left(\operatorname{diff}\left(c\left(\sigma\left(A_{n+1}(x)\right)\right), c\left(a_{1}\right)\right)\right)\right) \\
& =A_{n}\left(\bar{c}\left(\operatorname{diff}\left(c\left(A_{n+1}(x)\right)+1,1\right)\right)\right) \\
& =A_{n}\left(\bar{c}\left(c\left(A_{n+1}(x)\right)\right)\right)=A_{n}\left(A_{n+1}(x)\right) \\
& =A_{n+1}(\sigma(x)) .
\end{aligned}
$$

Using now definition 2, lemma 7 , the induction hypothesis and the relations

$$
f(x)=A_{n+1}(\pi(x)) \leq A_{n+1}(x) \leq A_{n+k+1}(x), x \in A^{*},
$$

we deduce that $A_{n+1}$ is in $C_{n+k+1}$ being obtained from functions belonging to $C_{n+k+1}$, using composition, limited iteration and $s(d i f f)$.

Lemma 8 For all naturals $n$ and all functions $f$ in $C_{n}$, there exists a natural $k$ such that $f(x)<A_{n}^{k}(x)$, for every string $x$ in $A^{*}$.

Proof. We shall make use of the inductive definition of $C_{n}$.

If $f(x)=A_{0}(x)$ then

$$
f(x)<A_{0}\left(A_{0}(x)\right) \leq A_{n}\left(A_{n}(x)\right)
$$

and we can take $k=2$.

If $f(x)=A_{n}(x)$, then

$$
f(x) \leq A_{n}\left(A_{n}(x)\right)
$$


and we can also take $k=2$.

If $f(x)<A_{n}^{p}(x)$ and $g(x)<A_{n}^{q}(x)$, for all strings $x$ in $A^{*}$ then

$$
\begin{aligned}
(f \circ g)(x) & \left.=f(g(x))<A_{n}^{p}(g(x))<A_{n}^{p+q}(x)\right), \\
s(\text { diff })(f, g)(x) & \leq f(x)<A_{n}^{p}(x) .
\end{aligned}
$$

Finally, if $f$ is obtained by limited iteration from $g$ and $h, h(x)<A_{n}^{k}(x)$, then $f(x) \leq h(x)<A_{n}^{k}(x)$.

Theorem 2 For every class $C_{n}, n \geq 1$, and every $f$ in $C_{n}$, there exists a natural $i$ (depending upon $f$ ) such that $f(x)<A_{n+1}(x)$ for every string $x$ in $A^{*}$ satisfying $c(x) \geq i$.

Proof. Assume that $f$ is a function in $C_{n}, n \geq 1$. In view of lemma 8 , we can find a natural $k \geq 2$ (which depends upon $f$ ) such that, for every string $x, f(x)<A_{n}^{k}(x)$. We shall show that the requested inequality holds for $i=3 k$.

From the monotonicity properties of Ackermann-Peter string-function, one can deduce the following relations:

$$
A_{n}^{k}(x)=A_{n}^{k-1}\left(A_{n}(x)\right) \leq A_{n}^{k-1}\left(A_{n}\left(\sigma^{k-1}(x)\right)\right)<A_{n}^{k-1}\left(A_{n+1}\left(\pi^{k-1}(x)\right)\right),
$$

for every string $x$ with $c(x)>3 k-1$.

Intermediate step: $A_{n+1}(x)=A_{n}^{k-1}\left(A_{n+1}\left(\pi^{k-1}(x)\right)\right)$, for every string $x$ with $c(x) \geq k$.

We shall prove the equality by induction on $x$. If $c(x)=k$, then we have

$$
\begin{aligned}
A_{n}^{k-1}\left(A_{n+1}\left(\pi^{k-1}(x)\right)\right) & =A_{n}^{k-1}\left(A_{n+1}\left(\pi^{k-1}\left(\sigma^{c(x)}(e)\right)\right)\right) \\
& =A_{n}^{k-1}\left(A_{n+1}\left(\pi^{k-1}\left(\sigma^{k}(e)\right)\right)\right)=A_{n}^{k-1}\left(A_{n+1}\left(a_{1}\right)\right) \\
& =A_{n}^{k-1}\left(A_{n}^{2}\left(a_{1}\right)\right)=A_{n}^{k+1}\left(a_{1}\right)=A_{n}^{c(x)+1}\left(a_{1}\right) \\
& =A_{n+1}(x) .
\end{aligned}
$$

If the equality holds for $x$, we can prove that

$$
A_{n+1}(\sigma(x))=A_{n}^{k-1}\left(A_{n+1}\left(\pi^{k-1}(\sigma(x))\right)\right) .
$$

Indeed,

$$
\begin{aligned}
A_{n}^{k-1}\left(A_{n+1}\left(\pi^{k-1}(\sigma(x))\right)\right) & =A_{n}^{k-1}\left(A_{n+1}\left(\sigma\left(\pi^{k-1}(x)\right)\right)\right) \\
& =A_{n}^{k-1}\left(A_{n}\left(A_{n+1}\left(\pi^{k-1}(x)\right)\right)\right) \\
& =A_{n}\left(A_{n}^{k-1}\left(A_{n+1}\left(\pi^{k-1}(x)\right)\right)\right) \\
& =A_{n}\left(A_{n+1}(x)\right)=A_{n+1}(\sigma(x)),
\end{aligned}
$$

and the intermediate step is proved.

Returning to the proof of the theorem, we can now write

$$
f(x)<A_{n}^{k}(x)<A_{n}^{k-1}\left(A_{n+1}\left(\pi^{k-1}(x)\right)\right)=A_{n+1}(x),
$$

for all strings $x$ with $c(x) \geq 3 k-1$ and taking $i=3 k-1$, the proof is finished. 
Theorem 3 The set $\bigcup_{n=0}^{\infty} C_{n}$ coincides with the set of unery primitive recursive string-functions.

Proof. We shall make use of the characterization of the set of unary primitive recursive string-functions obtained in [5], namely as the smallest class of unary string-functions which contains $\sigma$ and is closed under the operations

$$
s u b, \sigma-i t_{A, e}, s(\text { diff }) \text {. }
$$

It is obvious that every function in $\bigcup_{n=0}^{\infty} C_{n}$ is primitive recursive. For the converse inclusion, all that remains to be proved is reduced to the closure of $\bigcup_{n=0}^{\infty} C_{n}$ to $\sigma-i t_{A, \mathrm{e}}$.

We shall show that if $f \in \bigcup_{n=0}^{\infty} C_{n}$ is obtained by pure iteration from $g \in$ $\bigcup_{n=0}^{\infty} C_{n}$, there exists a function $h \in \bigcup_{n=0}^{\infty} C_{n}$ such that $f$ is obtained by limited iteration from $g$ and $h$ and, therefore, $f$ is in $\bigcup_{n=0}^{\infty} C_{n}$.

Indeed, let $f$ be obtained by pure iteration from $g$ in $C_{m}, m>0$. We shall prove, by induction on the string $x$ that $f$ is majorated by $A_{n+1}$.

If $x=e$, we have $f(e)=e<A_{n+1}(e)$.

Supposing that $f(x)<A_{n+1}(x)$ and using the definition and the monotonicity properties of Ackermann-Peter function, we get:

$$
f(\sigma(x))=g(f(x))<A_{n}(f(x))<A_{n}\left(A_{n+1}(x)\right)=A_{n}(\sigma(x)) .
$$

Theorem 4 The function $\bar{A}: A^{*} \longrightarrow A^{*}$ defined by $\bar{A}(w)=A_{c(w)}(w)$ is not primitive recursive.

Proof. Assume, on the contrary, that $\bar{A}$ is primitive recursive. From theorem 3 we get a natural $n$ such that $\bar{A} \in C_{n}$. By theorem 2 , there exists a natural $i$ such that $A(x)<A_{n+1}(x)$ for every $x$ with $c(x) \geq i$. Let $x$ be a string satisfying the condition $c(x)=n+i+1$. We arrive at a contradiction since

$$
\bar{A}(x)=A_{c(x)}(x)=A_{n+i+1}(x)<A_{n+1}(x)
$$

(see corollary 2). This completes the proof of the theorem.

\section{Acknowledgements}

We are grateful to Dr. Cristian Calude for drawing our attention to these problems and for many helpful remarks.

\section{References}

[1] G.Asser. Rekursive Wortfunktionen Z.Math. Logik Grundlag.Math. 6(1960), 258-278. 
[2] G.Asser. Primitive recursive word-functions of one variable, in E.Borger (ed.), Computation Theory and Logic, LNCS 270, Springer 1987, 14-19.

[3] G.Asser. Zur Robinson Charakterisierung der Einstelligen Primitiv Rekursiven Wortfunktionen, Z.Math.Logik Grundlag.Math., 34(1988), 317-322.

[4] C.Calude. Theories of Computational Complexity, North-Holland, Amsterdam, New-York, Oxford, Tokio, 1988.

[5] C.Calude, L.Santean. On a Theorem of Gunter Asser, Z.Math.Logik Grundlag.Math., 1990.

[6] F.W.v.Henke, K.Indermark, G.Rose, K.Weichrauch. On Primitive Recursive Wordfunctions, Computing 15(1975), 217-234.

[7] M.Tatarim. Darboux property and one-argument primitive recursive stringfunctions, Revue Roumaine des Mathematiques Pures et Appliques, 1987, 7994.

[8] K.Weichrauch. Teilklassen primitiv-rekursiver Wortfunktionen, Berichte der GMD 91(1974), 1-49. 